\title{
Special issue on Web information management and applications
}

\author{
Yi Cai ${ }^{1} \cdot$ Jianliang $\mathrm{Xu}^{2}$
}

Published online: 15 February 2020

(C) Springer Science+Business Media, LLC, part of Springer Nature 2020

\section{APWeb-WAIM 2018}

We are pleased to present a special issue of World Wide Web Journal (WWWJ), which contains a collection of three extended papers from the APWeb-WAIM 2018 conference.

APWeb-WAIM conferences focus on research, development, and applications in relation to Web information management, including a wide range of topics, such as text analysis, graph data processing, social networks, recommender systems, information retrieval, data streams, knowledge graph, data mining and application, query processing, machine learning, database and Web applications, big data, and blockchain. APWeb-WAIM 2018 was held in Macau during July 23-25, 2018, and attracted a total of 168 research paper submissions. The conference program committee selected 39 full research papers, 31 short papers, and six demonstration papers to be presented at the conference and published in the conference proceedings $[1,2]$. The conference program also included keynote presentations by Prof. Xuemin Lin (The University of New South Wales, Australia), Prof. Lei Chen (The Hong Kong University of Science and Technology, Hong Kong, SAR China), and Prof. Ninghui Li (Purdue University, USA) as well as industrial invited talks by Dr. Zhao Cao (Huawei Blockchain) and Jun Yan (YiDu Cloud).

\section{The special issues}

The three extended papers for this special issue were selected from among all the accepted papers by the special issue guest editors Yi Cai and Jianliang Xu, based on the relevance to the journal and the reviews of the conference version of the papers. The authors were asked to

\section{Yi Cai}

ycai@scut.edu.cn

Jianliang Xu

xuj1@Comp.HKBU.Edu.HK

1 School of Software Engineering, South China University of Technology, Guangzhou, China

2 Department of Computer Science, Hong Kong Baptist University, Kowloon Tong, Hong Kong, China 
revise the conference paper for journal publication and in accordance with customary practice of adding $70 \%$ new material. The revised papers again went through the standard review process in accordance with WWWJ guidelines and are finally presented to the readers in the present form.

The three extended papers in this special issue cover a variety of topics related to Web information management and applications. In the first paper, "User Experience-Driven Secure Task Assignment in Spatial Crowdsourcing," authors define user experience-driven secure task assignment problem and propose two privacy-preserving online task assignment strategies to minimize the average waiting time. The second paper, "Personalized Top-n Influential Community Search over Large Social Networks," presents a maximal pk-Clique community model by a new cohesive criterion and proposes a diversify algorithm which is based on a tree-like index. Finally, in "An Enhanced Wildcard-based Fuzzy Searching Scheme in Encrypted Databases," authors present an enhanced wildcard-based fuzzy searching scheme (enWFS) to explore more business logic in encrypted databases, and also design an advanced adaptive overwriting method to revise query expressions with wildcards.

Acknowledgements We would like to acknowledge the work done by all authors and their willingness to contribute their papers for this special issue. We thank all the reviewers for their expert comments and assistance in timely reviews. Special thanks to the journal editors for their great help and support in organizing the issue.

\section{References}

1. Cai Y, Ishikawa Y, Xu J (2018) Web and big data-second international joint conference, APWeb-WAIM 2018, Macau, China, July 23-25, 2018, Proceedings, Part I. Lecture Notes in Computer Science 10987, Springer 2018. ISBN: 978-3-319-96889-6

2. Cai Y, Ishikawa Y, Xu J (2018) Web and big data - second international joint conference, APWeb-WAIM 2018, Macau, China, July 23-25, 2018, Proceedings, Part II. Lecture Notes in Computer Science 10988, Springer 2018. ISBN: 978-3-319-96892-6

Publisher's note Springer Nature remains neutral with regard to jurisdictional claims in published maps and institutional affiliations. 